\title{
Simulation of BPMN Process Models: Current BPM Tools Capabilities
}

\author{
José Luís Pereira $^{1}$ and António Paulo Freitas ${ }^{1}$, \\ ${ }^{1}$ Universidade do Minho, Departamento de Sistemas de Informação, \\ Campus de Azurém, Guimarães, Portugal \\ jlmp@dsi.uminho.pt,a59742@alunos.uminho.pt
}

\begin{abstract}
Nowadays, more and more organizations are using Business Process Management (BPM) to prepare themselves to deal, in an effective way, with the increasingly difficult conditions of modern markets. Essential to BPM is the collection of tools which support the operationalization of the business process concept - BPM tools. These tools deal with business process models, which have to be described with a suitable language. In the present, BPMN is considered the standard modeling language to describe business processes. Once a business process is modeled, a process simulation approach might be used in order to find its optimized version. Therefore, the simulation of business process models, such as those defined in BPMN, appears as an obvious way of improving processes. This paper advances work previously published by the authors regarding BPM tools capabilities in terms of the simulation of BPMN process models. In this context a platform to support the characterization of BPM tools regarding process simulation capabilities has been developed. This platform might be helpful to users who want to select the most adequate BPM tool regarding their simulation needs.
\end{abstract}

Keywords: BPM tools, BPMN, process modeling, process simulation.

\section{Introduction}

Nowadays, more and more organizations are using Business Process Management (BPM) to prepare themselves to deal, in an effective way, with the increasingly difficult conditions of modern markets. Indeed, it is widely recognized that a business processes oriented management provides organizations with increased levels of performance and flexibility, as they can respond to the needs and changes of the markets in a most efficient and effective way [1].

Essential to BPM is the collection of tools which support the operationalization of the business process concept - the BPM tools. Due to the increasing relevance of the worldwide BPM market, BPM tools producers are improving their tools in order to gain higher market acceptance [2].

Core to BPM tools is the concept of business process model, which has to be described with a suitable language. Regarding the modeling of business processes there are several languages in use today, such as BPMN (Business Process Model and 
Notation) [3], EPC (Event-driven Process Chain) [4], or UML-AD (Unified Modeling Language - Activity Diagrams) [5], to name just a few.

Since its conception, the BPMN has gained worldwide acceptance, and is now recognized as the standard process modeling language to use in the development of BPM projects. The use of this language has simplified the way organizations represent and communicate their business processes, as BPMN allows business process modelers to represent complex business processes easily and effectively [3].

The modeling of business processes, using the BPMN language, allows organizations to obtain graphical representations of their processes. Using the produced diagrams (Business Process Diagrams), organizations can assess whether their processes present anomalies, inconsistencies, inefficiencies and, therefore, improvement opportunities. The inability to quantify the processes weaknesses can be eliminated by organizations through the use of simulation. This approach allows organizations to anticipate process behaviors, based on estimations and mathematical calculations performed with the aid of a computer, thus letting them identify and quantify its shortcomings and anomalies.

This paper advances work previously published by the authors regarding BPM tools capabilities in terms of the simulation of BPMN process models. In a previous paper [6], we have analyzed the business process modeling and simulation areas, to identify the elements that must be present in the BPMN language in order to allow processes described in BPMN to be simulated. We concluded that, although there are several BPM tools with simulation capabilities which support BPMN, they still present several limitations regarding the simulation of process models. In this context, a platform to support the characterization of BPM tools regarding process simulation capabilities has been developed. This platform, which is completely extensible in terms of the characterized BPM tools and simulation properties to be supported, might be helpful to users who want to select the most adequate BPM tool regarding their simulation needs.

Concerning the structure of this paper, first we very briefly mention the BPMN language. Next, we identify the elements that the BPMN language and BPM tools have to incorporate in order to enable the simulation of business processes simulation properties. In the following, we present the results of the analysis we made to some well-known BPM tools, regarding their support of those simulation properties. These results constitute the initial base of the platform we have developed to characterize BPM tools simulation capabilities, which is prepared to incorporate other evaluations, of the same or other BPM tools, using the same or other simulation properties. The core functionalities of this platform are also presented.

\section{The BPMN Language}

Before a business process can be analyzed, optimized, implemented and managed, it has to be modeled. Models of business processes are developed using specific languages, of which BPMN is one of the most widely used. BPMN stands for Business Process Model and Notation and is a language that has appeared in 2004, developed and sponsored by the Business Process Management Initiative (BPMI), 
and later adopted as standard by the Object Management Group (OMG). The first version of BPMN was developed in order to standardize the graphical representation of business processes, providing a set of "graphic symbols" for the various elements of the process, with a coherent meaning and ability to represent their possible combinations [7].

White [8] argues that the main objective of BPMN is to provide a notation that is understandable to all the stakeholders around organizational processes, from business analysts, who document or define the models of business processes, to technical developers, who are responsible for developing the IT solutions that will support the those processes, and finally, to all users who will control and manage the processes developed.

The notation for the graphical representation of the language elements was designed so that different elements are distinguishable from each other and noticeable for modelers. For instance, it is normal to associate rectangles to business process activities, while decisions are represented by diamonds [9].

To organize the graphic elements of the language, BPMN distinguishes among five specific categories (flow objects, data, connection objects, swimlanes and artifacts). The flow objects, which are the major graphic elements to represent the behavior of a business process, are divided into three groups (events, activities and gateways). The data category provides the information necessary for the activities, and is divided into four groups (data object, collections of data, input data and output data). The connection objects define the way objects are linked and the order in which activities are performed during the process. Currently there are three groups of connection objects (sequence flows, message flows and associations). Swimlanes are divided in two categories (pool and lane). Pools allow the identification of the actors involved in the process. In order to increase the detail, pools may be sub-divided in lanes. Artifacts are used in order to provide additional information about the process that is represented. Currently, there are two types of artifacts (group and notes), and the process modeler can add more than one artifact to the process model.

\section{BPM Tools and Process Simulation with BPMN}

It is widely acknowledged today that simulation experiments are a reliable and credible source of insights with regard to the support of decision-making in organizations. Indeed, the ability to anticipate, in a tangible and understandable way, the probable results of a decision before making it in the real world, allows managers to better ground their decisions. Simply put, simulation assists managers in their decision-making duties, since it allows them to develop and analyze various scenarios of possible interest. The use of simulation to analyze "what if" scenarios eliminates the costs and risks that are inherent to testing them in a real environment.

As Sakurada \& Miyake [10] put it, simulation is a technique that may be applied to understand the behavior of a system, with the purpose of assessing the consequences arising from changes made to their processes and/or physical settings (e.g. the capacity of the resources to execute tasks and the final aspect of the facility), without any disturbances to current operations. 
With the growing importance of BPM in organizations, the use of BPMN to model their business processes is gaining more and more followers. If the elements needed for the simulation of processes were incorporated into the BPMN language, then one would be able, not only to model the processes, but also to simulate them. That is, by using the BPMN language enriched with the proper elements needed to support simulation, users not only have the ability to model business processes, but also the ability to simulate the processes modeled, with all the advantages of testing and analyzing different scenarios without any risks to the real system [11].

BPMN has been designed to standardize the graphical representation of business processes, without any concerns about simulation. Therefore, there is a need to define a set of "extensions" to the BPMN language, in order to allow process models developed in BPMN to be simulated. These are properties that the proponents of the BPMN language did not anticipate, but that are essential, so process models can be simulated.

In [6] we have identified a set of simulation properties grouped into six classes: Context Definition, Time Consumption, Control, Resources, Costs and Priorities (see Table 1). Some of these properties might be reflected in the BPMN models, others relate to the simulation engines themselves.

Table 1. Simulation Properties.

\begin{tabular}{|c|c|}
\hline Properties & Description \\
\hline \multicolumn{2}{|l|}{ Context Definition } \\
\hline Starting Time & Setting a start time to run the simulation \\
\hline Duration & Setting the duration of the simulation \\
\hline Time Unit & Defining the time unit of the simulation \\
\hline Cost Unit & Defining the cost unit of the simulation \\
\hline Replications & Number of replications of the simulation \\
\hline \multicolumn{2}{|l|}{ Time Consumption } \\
\hline Transfer Time & Time spent in transit from one activity to the next \\
\hline Waiting Time & Time spent waiting to be executed (queue time) \\
\hline Processing Time & Time spent in the execution of an activity (probability distribution) \\
\hline \multicolumn{2}{|l|}{ Control } \\
\hline Arrival Rate & Definition of the process triggering pattern (probability distribution) \\
\hline Branch Probabilities & Definition of probabilities for each branch out of a gateway \\
\hline \multicolumn{2}{|l|}{ Resources } \\
\hline Capacity & Setting the number of resources available to execute each activity \\
\hline Allocation Plan & Definition of a sharing plan for the execution of activities \\
\hline Unavailability & Definition of unavailability periods for resources \\
\hline Schedule & Definition of work schedules for resources \\
\hline \multicolumn{2}{|l|}{ Costs } \\
\hline Activity & Definition of the processing cost of an activity \\
\hline Resource & Definition of the hourly cost of each resource \\
\hline \multicolumn{2}{|l|}{ Priorities } \\
\hline Interruptions & Definition of activities that cannot be interrupted while running \\
\hline Execution Priority & Definition of activities that have priority in execution \\
\hline
\end{tabular}


Concerning probability distributions, there are several distributions, which are normally available in every simulation tool (such as Normal, Triangular, Uniform, Beta, Exponential, Gamma, Erlang, Binomial, and Poisson), that should also be present in a BPM tool with simulation capabilities.

\section{BPM Tools Analysis Regarding Process Simulation}

Until recently, to simulate a business process modeled in, for instance BPMN, an analyst had to re-model the process model according to the specific language of the selected simulation tool. Such a situation is unjustified and awkward as it involves a duplication of work. Fortunately, this situation has begun to change, as more and more tools emerge in the market, which allow the simulation of business processes modeled in BPMN.

However, one can find a common pattern among those tools - all of them originated from BPM tools vendors, not from simulation tools vendors. So, these are essentially BPM tools which have been extended with simulation capabilities, not simulation tools which have incorporated BPMN as a modeling language. Therefore, it is not clear right now if those tools can, effectively, simulate BPMN business processes with the desired level of realism and accuracy. In order to clarify this situation we have made an analysis of the following tools, which have made relevant incursions in the simulation area, taking into consideration the simulation properties identified in Table 1:

- $\quad$ Bizagi (Modeler version 2.9);

- BIMP (online version);

- BonitaSoft (version 6.5.3);

- Visual Paradigm (version 12.1);

- BPSim (Trisotech BPMN 2.0 Modeler for Visio version 4.2.0).

To facilitate the analysis of these tools a simple scenario was developed which uses a common business process ("Request of Order Delivery Date"), dealing with the approval of the delivery date of an order. The main goal was to assess, for each BPM tool, which simulation properties are present or absent. The next table (Table 2) is a summarization of the simulation capabilities of each tool, taking into account the properties previously identified in Table 1. A mark (X) signals that a property is supported by the tool.

Table 2. Simulation Properties by Tool.

\begin{tabular}{|c|c|c|c|c|c|}
\hline Properties & BIMP & Bizagi & BPSim & BonitaSoft & $\begin{array}{c}\text { Visual } \\
\text { Paradigm }\end{array}$ \\
\hline \multicolumn{6}{|c|}{ Context Definition } \\
\hline Starting Time & $\mathrm{X}$ & $\mathrm{X}$ & $X$ & $X$ & $X$ \\
\hline Duration & $X$ & $X$ & $\mathrm{X}$ & $\mathrm{X}$ & $X$ \\
\hline Time Unit & $\mathrm{X}$ & $X$ & $X$ & $X$ & $X$ \\
\hline Cost Unit & $\mathrm{X}$ & $X$ & $X$ & $\mathrm{X}$ & $\mathrm{X}$ \\
\hline Replications & & $\mathrm{X}$ & $X$ & & \\
\hline
\end{tabular}




\begin{tabular}{|c|c|c|c|c|c|}
\hline \multicolumn{6}{|l|}{ Time Consumption } \\
\hline Transfer Time & & & $\mathrm{X}$ & & \\
\hline Waiting Time & $X$ & $\mathrm{X}$ & $X$ & $\mathrm{X}$ & $\mathrm{X}$ \\
\hline Processing Time & $X$ & $X$ & $X$ & $X$ & $\mathrm{X}$ \\
\hline \multicolumn{6}{|l|}{ Control } \\
\hline Arrival Rate & $\mathrm{X}$ & $\mathrm{X}$ & $\mathrm{X}$ & $\mathrm{X}$ & \\
\hline Branch Probabilities & $X$ & $\mathrm{X}$ & $\mathrm{X}$ & & $\mathrm{X}$ \\
\hline \multicolumn{6}{|l|}{ Resources } \\
\hline Capacity & $X$ & $\mathrm{X}$ & $X$ & $X$ & $\mathrm{X}$ \\
\hline Allocation Plan & & $\mathrm{X}$ & $\mathrm{X}$ & & \\
\hline \multicolumn{6}{|l|}{ Unavailability } \\
\hline Schedule & $\mathrm{X}$ & $\mathrm{X}$ & $X$ & $X$ & \\
\hline \multicolumn{6}{|l|}{ Costs } \\
\hline Activity & $\mathrm{X}$ & $\mathrm{X}$ & $\mathrm{X}$ & $\mathrm{X}$ & $\mathrm{X}$ \\
\hline Resource & $\mathrm{X}$ & $\mathrm{X}$ & $\mathrm{X}$ & $X$ & \\
\hline \multicolumn{6}{|l|}{ Priorities } \\
\hline Interruptions & & & $\mathrm{X}$ & & \\
\hline Execution Priority & & & $\mathrm{X}$ & & \\
\hline \multicolumn{6}{|l|}{ Probability Distribution } \\
\hline Normal & $\mathrm{X}$ & $\mathrm{X}$ & $X$ & $X$ & $\mathrm{X}$ \\
\hline Triangular & $X$ & $\mathrm{X}$ & $X$ & & \\
\hline Uniform & $\mathrm{X}$ & $\mathrm{X}$ & $\mathrm{X}$ & & \\
\hline Beta & & $\mathrm{X}$ & $\mathrm{X}$ & & \\
\hline Erlang & & $\mathrm{X}$ & $\mathrm{X}$ & & \\
\hline Poisson & & $X$ & $X$ & & \\
\hline
\end{tabular}

As we can see, there are substantial differences among tools regarding simulation capabilities. Considering that a simulation experiment is intended to faithfully represent the real system or, at least, represent it as accurately as possible, BPM tools that have limitations concerning the support of simulation properties won't be able to fulfill that requirement, leading to simulation results that might be far away from the real ones.

\section{BPM Tools Simulation Capabilities Platform Development}

Based on the identified groups of simulation properties that were identified (Table 1) we decided to develop a platform to support the characterization of BPM tools regarding their capabilities in terms of simulation support.

At this moment, a functional prototype of the platform, whose user interface, for now, is mainly in Portuguese, has already been developed. We decided to create two versions of the platform: a desktop version, with all the functionalities described below; and a web version, with a more limited set of functionalities (in particular, the extensibility functionalities), but with higher potential to reach more users, as soon as we translate the user interface to English. In the rest of this section, we choose to present only parts of the desktop version of the platform. 
In Table 2, one can see the results obtained with our analysis of the five tools selected to this study. In that table we only show if a simulation property is, or is not, supported by a tool. Of course, a simple indication of presence/absence is not enough to properly characterize a given tool, as different tools may support the same simulation property with distinct levels of support. To solve that problem, if a tool supports a property, we propose to quantitatively characterize the level of support in a scale from 1 to 5 (1 - Insufficient;...; 5 - Excellent).

Besides a quantification of the support level to characterize how a given tool supports a given simulation property, there are other requirements that this platform must fulfill, in order to be useful.

One of the requirements we think is of utmost importance is that the platform should have the capacity to evolve. The platform should be extensible in the sense that the set of BPM tools could be expanded, and also the list of groups and simulation properties could be extended, if needed. In Fig. 1 we show some of the mockups used to maintain the set of BPM tools, groups of simulation properties, and simulation properties.

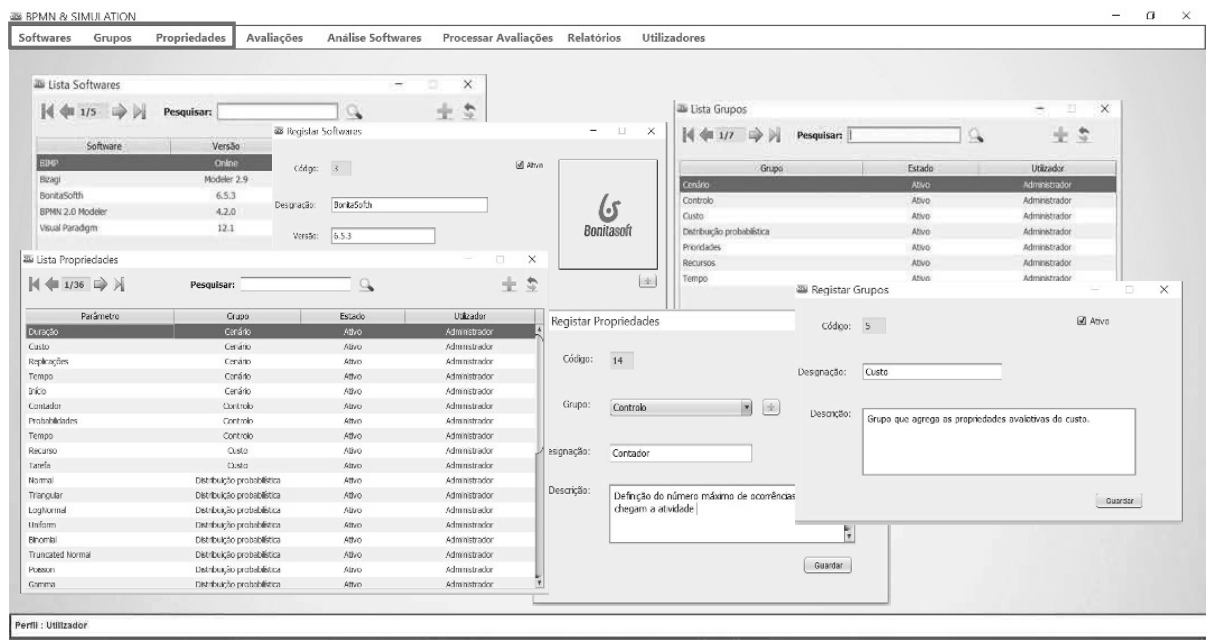

Fig. 1. Platform Maintenance - BPM Tools, Groups of Properties and Simulation Properties

The quantification of the support levels must be updated dynamically, taking into consideration the opinions of other BPM tool users. Indeed, it would be convenient that the contents of the platform could evolve by incorporating the evaluations of experienced users (contributors), thus improving its accuracy. In that sense, at each moment, the "knowledge" present in the platform would represent the weighted evaluations of the users that have contributed to its content until that moment.

If a contributor wants to make an evaluation, he/she has to select the BPM tool to characterize, the property groups to evaluate and, for each one of the selected groups, the properties to evaluate (Fig. 2). A contributor might also desire to change an evaluation that he/she has done in the past. Of course, this is also possible, as users change their opinions about BPM tools, and BPM tools also evolve thus leading to the need to change evaluations. 


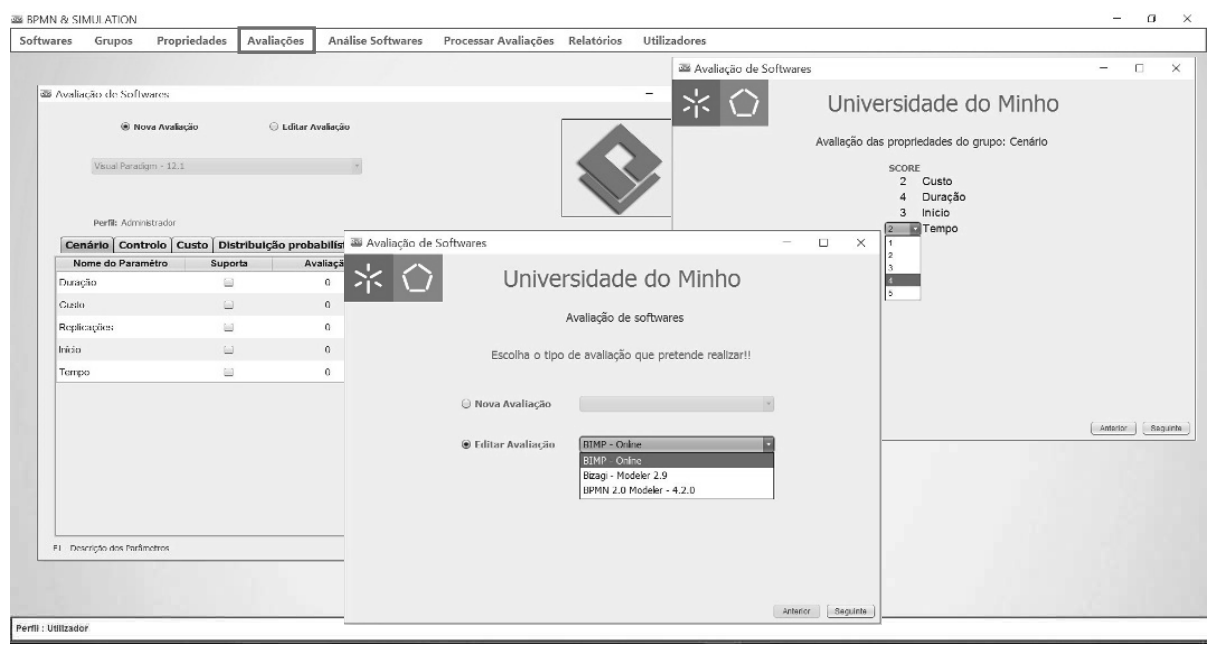

Fig. 2. Platform Maintenance - Evaluation of BPM Tools

Finally, the platform should assist users in the comparison of BPM tools, taking into account their specific needs in terms of simulation support. To do that, users should have the possibility to give weights to each of the simulation properties, in order to best characterize their needs in terms of simulation. So, users select the BPM tools and the groups of simulation properties, in which they are interested. For each group they select the desired simulation properties, assigning a value to each one of them (in a scale from 1 to 5), thus quantifying their interest in them (Fig. 3).

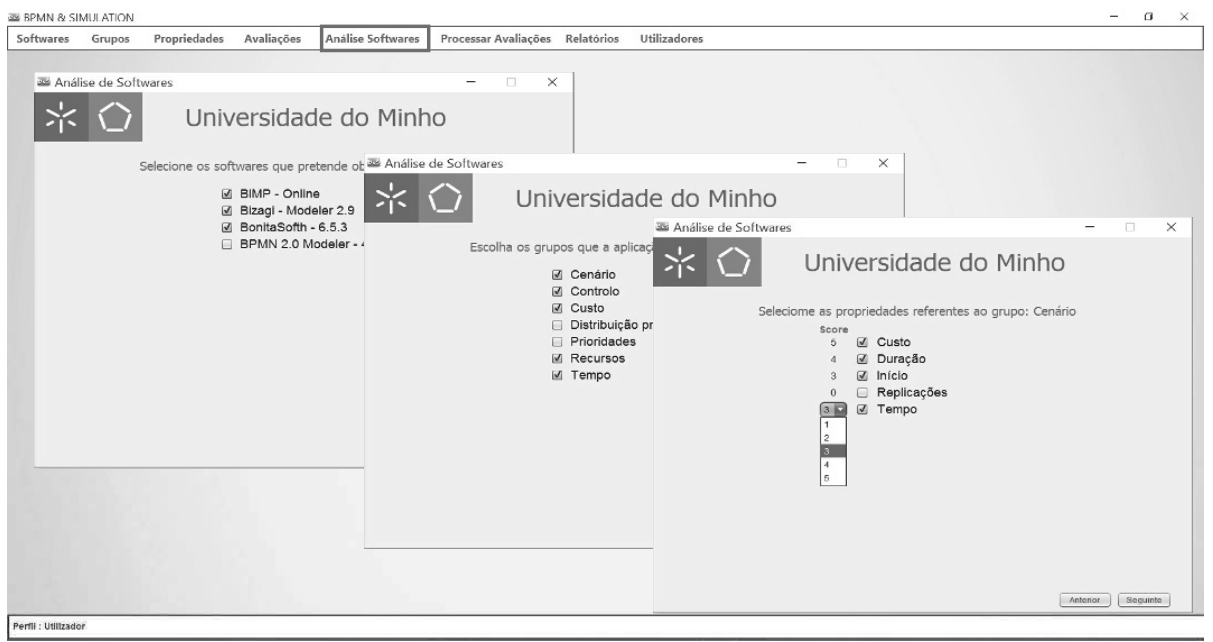

Fig. 3. Selection of BPM Tools, Groups of Properties and Simulation Properties to Analyze

Thus, obtaining the required information in the form of several different graphics and reports that he may choose (Fig. 4). 


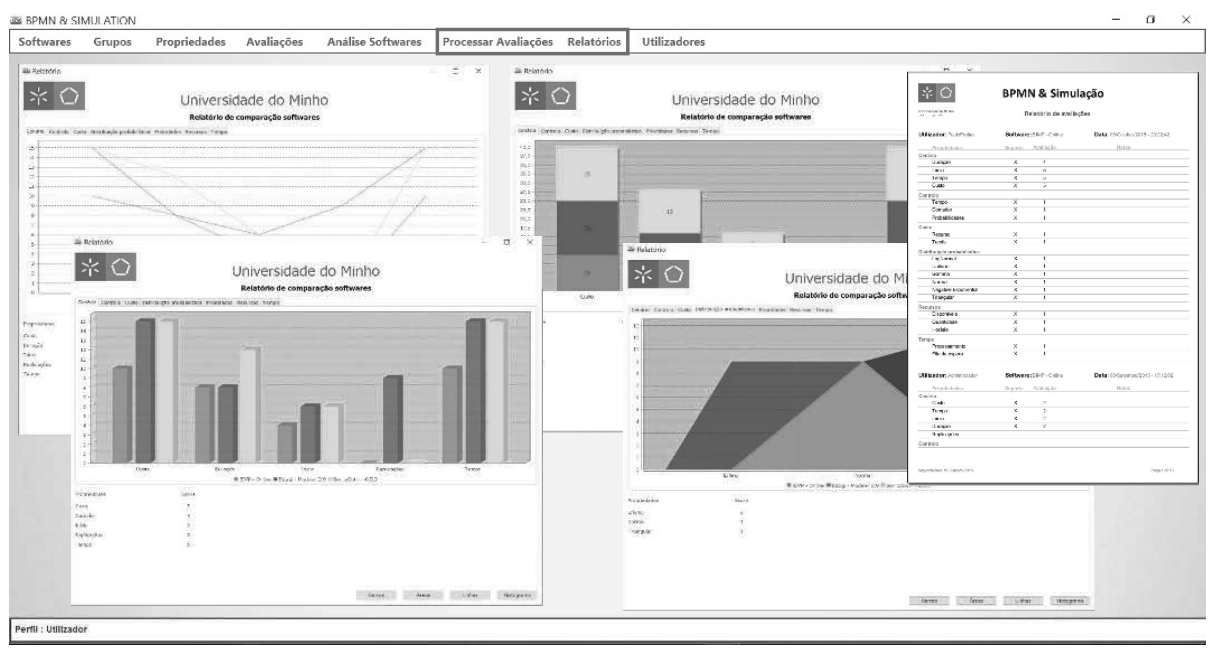

Fig. 4. Results of the BPM Tools Comparison

As was mentioned before, at this moment the user interface of the platform is entirely in the Portuguese language which, at least in the case of the web version, is an important limitation to its more ample usage. In the near future we intend to translate all the user interface to English, in order to make the platform available to more users.

\section{Conclusions}

With the worldwide acceptance of the BPM (Business Process Management) approach by organizations, a class of software tools has gained relevance - BPM tools. This is a large family of products intended to support the needs of organizations regarding the modeling, implementation, execution, monitoring and improvement of their business processes.

In this paper, we focus our attention, once again, in the modeling of business processes using the widely used business process modeling language BPMN. More specifically we want to clarify which is the level of support offered by modern BPM tools, regarding the simulation of business processes modeled in BPMN. Our interest in process simulation comes from the fact that the ability to simulate business processes, before their actual implementation in the field, might provide substantial gains to organizations and reduce the risks associated to changes. Indeed, by using simulation organizations may anticipate the effects of changes in their processes and evaluate different scenarios (what-if).

The analysis of some BPM tools underlying this work has revealed that, besides a minimum set of features needed to do simple simulation work, and which is common to all of them, there are very distinct simulation capabilities among tools. On the one hand, while there are already some BPM tools which may be used to do serious simulation work, on the other hand, there are others that still lack essential simulation capabilities. Anyway, it became clear that there is already an effort from BPM tools 
developers, particularly those that support the BPMN standard, in enabling their products with simulation features. One can expect that, in a few years, simulation capabilities will be standard features of BPM tools.

This paper complements previous published work from the same authors with the presentation of a platform that was developed to support the characterization of BPM tools regarding their simulation capabilities. The prototype of the platform, which is available as a desktop application and as a web portal, is open to the community of BPM tools users, waiting to receive their contributions in order to improve its contents. The platform is prepared to continuously evolve and improve its content, giving support to users who need to choose a BPM tool, taking into account their specific simulation needs.

\section{References}

1. Brocke, J., \& Rosemann, M. (2010). Handbook on Business Process Management 2. Decision Support Systems.

2. Buelow, H. (2010). Getting Started with Oracle BPM Suite 11gR1. Packt Publishing Ltd.

3. Freund, J. \& Rücker, B. (2014). Real-Life BPMN: Using BPMN 2.0 to Analyze, Improve, and Automate Processes in Your Company (2nd Ed), CreateSpace Independent Publishing Platform.

4. Davis, R. (2008). ARIS Design Platform: Advanced Process Modelling and Administration, Springer.

5. Podeswa, H. (2009). UML for the IT Business Analyst (2nd Ed), Cengage Learning PTR.

6. Freitas, A.P., \& Pereira. J.L. (2015). Process Simulation Support in BPM Tools: The Case of BPMN. Proceedings of the 5th International Conference on Business Sustainability. Póvoa de Varzim - Portugal.

7. OMG. (2011). Business Process Model and Notation (BPMN). Object Management Group, Inc.

8. White, S. a. (2004a). Business process modeling notation. Specification, BPMI. Org, (January), 1-296.

9. White, S. a. (2004b). Introduction to BPMN. BPTrends, 1-11.

10.Sakurada, N., \& Miyake, D. (2009). Simulação baseada em agentes (SBA) para modelagem de sistema de operações. Simpoi, ANAIS (1980), 1-16.

11.Ribeiro, M., \& Pereira. J. (2014). Multi-paradigm simulation projects: The need for practical guidelines. Proceedings of the 4th International Conference on Business Sustainability. BS 2014. Póvoa de Varzim - Portugal.

12. Oliveira, P., \& Pereira, J. (2008). A Simulação Computorizada no Suporte à Optimização e Melhoria Contínua de Processos Organizacionais. Proceedings da $8^{a}$ Conferência da Associação Portuguesa de Sistemas de Informação. CAPSI 2008. Setúbal - Portugal.

13.Waller, A., Clark, M., \& Enstone, L. (2006). L-SIM: Simulating BPMN Diagrams with a Purpose Built Engine, Proceedings of the Winter Simulation Conference. WSC 2006. 591597. Monterey, CA. 